\title{
Curcumin reverses benzidine-induced epithelial-mesenchymal transition via suppression of ERK5/AP-1 in SV-40 immortalized human urothelial cells
}

\author{
ZHIQI LIU ${ }^{1 *}$, JIE LIU $^{1 *}$, LI ZHAO $^{1}$, HAO GENG $^{1}$, JIAXING MA ${ }^{1}$, \\ ZHIQIANG ZHANG ${ }^{1}$, DEXIN YU ${ }^{1}$ and CAIYUN ZHONG ${ }^{2}$ \\ ${ }^{1}$ Department of Urology, The Second Affiliated Hospital of Anhui Medical University, Hefei, Anhui 230032; \\ ${ }^{2}$ Department of Nutrition and Food Safety, School of Public Health, Nanjing Medical University, \\ Nanjing, Jiangsu 211166, P.R. China
}

Received September 4, 2016; Accepted December 22, 2016

DOI: 10.3892/ijo.2017.3887

\begin{abstract}
Overexposure to benzidine has been manifested as an important cause of bladder cancer. However, the molecular mechanism of benzidine-induced malignancy is still insufficiently interpreted. Epithelial-mesenchymal transition (EMT) is a crucial pathophysiological process in embryonic development as well as initiation and development of epithelium-originated malignant tumors. The role of extracellular regulated protein kinase 5 (ERK5) in benzidine-meditated bladder cancer development has not been explored. In the present study, we explored the role of ERK5/AP-1 pathway in benzidine-induced EMT in human normal urothelial cells and the intervention effect of curcumin on bezidine-induced EMT. We found that benzidine-induced EMT in SV-40 immortalized human urothelial cells (SV-HUC-1) at low concentrations. We detected that ERK5/AP-1 pathway was notably activated. Specific ERK5 inhibitor, XMD8-92 was applied to determine the role of ERK5 in benzidine-induced EMT. Results indicated that XMD8-92 reversed the EMT process. Furthermore, curcumin effectively attenuated benzidine-induced urocystic EMT by suppressing ERK5/AP-1 pathway. In conclusion, the present study revealed the positive role of ERK5/AP-1 in benzidine-provoked urocystic EMT and the curcumin promising use in bladder cancer prevention and intervention via ERK5/AP-1 pathway.
\end{abstract}

Correspondence to: Professor Dexin Yu, Department of Urology The Second Affiliated Hospital of Anhui Medical University, Hefei, Anhui 230032, P.R. China

E-mail: yudx_urology@126.com

Professor Caiyun Zhong, Department of Nutrition and Food Safety, School of Public Health, Nanjing Medical University, Nanjing, Jiangsu 211166, P.R. China

E-mail: cyzhong@njmu.edu.cn

*Contributed equally

Key words: benzidine, bladder cancer, ERK5/AP-1, curcumin, EMT

\section{Introduction}

Bladder cancer is the most common cancer of urinary tract with an estimation of approximately 80,500 new cases and 32,900 deaths and non-uniform distribution among urban and rural areas in China in 2015 (1). Epidemiologic studies revealed that cigarette smoking, exposure to arsenic and occupational exposure to aromatic amines are risk factors of bladder cancer (2).

Benzidine, also called 4,4'-diaminobiphenyl, is an aromatic amine, which has been classified as definite human carcinogen by the International Agency for Research on Cancer (IARC) with urinary bladder being a major target (3-5). Due to its wide distribution in the chemical, dye and rubber industries, cigarette smoke, automobile exhaust and industrial pollutant emissions, benzidine still is a great hazard to human health (6-8). Notwithstanding, there have been epidemiological research revealing the correlation between benzidine exposure and bladder oncogenesis, the molecular pathogenesis is still largely unknown.

Epithelial-mesenchymal transition (EMT) is a pathophysiological process that involves in multiple processes including embryogenesis, tissue reconstruction, organ fibrosis and cancer development. Epithelia lose epithelial characteristics and obtain mesenchymal traits via EMT, such as loss of cell polarity, enhancement of cell invasive and metastatic ability (9-11). Abundant evidence demonstrates the crucial role of EMT in the invasion and metastasis of cancer cells, including bladder cancer. Benzidine exposure has been manifested to induce EMT in urinary bladder (12). However, the molecular mechanism of benzidine-induced EMT remains insufficiently illuminated.

The mitogen-activated protein kinases (MAPKs) include four major subfamilies: extracellular signal-related kinases (ERK 1/2), Jun amino-terminal kinases (JNK1/2/3), p38 proteins $(\mathrm{p} 38 \alpha / \beta / \gamma / \delta)$ and ERK5 (13). ERK5 (also called big mitogen-activated protein kinase 1) (BMK1), is the least studied member of MAPK family. The MEK/ERK5 is involved in multiple important processes including cell differentiation and survival, cell proliferation, angiogenesis 
and tumorigenesis (14-17). Activated protein 1 (AP-1) is a heterodimer composed of Jun and Fos proteins. As a transcription activator, AP-1 acts in various cell activities. Compelling evidence shows that the mitogen activated protein kinase (MAPK)/AP-1 pathway plays an important role in cancer initiation and progression $(18,19)$. In a previous study, our groups revealed that activation of ERK5/AP-1 upregulates cigarette smoke induced EMT of normal urothelial cells (20), but investigations have not been made to detect the correlation between ERK5/AP-1 and benzidine exposure, and the role of ERK5 in benzidine-provoked EMT remains to be elucidated.

Curcumin is a polyphenol (1,7-bis(4-hydroxy-3-methoxyphenyl)-1,6-heptadiene-3,5-dione) with an outstanding safety profile and is the major bioactive component of turmeric, a spice commonly used as a food additive and a traditional herbal medicine with a long history throughout Asia (21). Former research demonstrated the anticancer activities of curcumin derivatives on a variety of cancers including lung, breast, colorectal and thyroid cancer in vitro and in vivo. However, few studies have been conducted to detect the interventional activities of curcumin on benzidine-related bladder EMT.

The aim of present study was to investigate the mechanism of benzidine induced malignancy in human normal urothelial cells as well as chemical preventive effect of curcumin on this process, which may provide new insight into benzidine-related carcinogenesis and cancer intervention.

\section{Materials and methods}

Chemicals and reagents. SV-40 immortalized human urothelial cell line (SV-HUC-1) was obtained from the Chinese Academy of Type Culture Collection Cell Bank (Shanghai, China). Curcumin was purchased from Sigma-Aldrich (St. Louis, MO, USA; purity, 99.0\%). Benzidine (4,4'-diaminobiphenyl; $\geq 98.0 \%$, RT), dimethyl sulfoxide (DMSO) and methanol were obtained from Merck (Reading Township, NJ, USA). Growth media (F-12K medium, Kaighn's Medium; Wisent Inc., Montreal, QC, Canada). Fetal bovine serum (FBS), phosphate-buffered saline (PBS), antibiotics and trypsin were obtained from HyClone Laboratories (Logan, UT, USA). 3-(4,5-Dimethylthiazol-2-yl)-2,5-diphenyltetrazolium bromide (MTT) was purchased from Sigma-Aldrich. Phosphorylated ERK5 (p-ERK5), phosphorylated c-jun (p-cjun), phosphorylated c-fos (p-c-fos), E-cadherin, N-cadherin and vimentin were obtained from Cell Signaling Technology (Beverly, MA, USA). The antibody for ZO-1 was obtained from Santa Cruz Biotechnology (Santa Cruz, CA, USA). The GAPDH antibody was obtained from Biogot Technology (Nanjing, China). XMD8-92 was purchased from Selleck Chemicals (Huston, TX, USA). Sources of other materials are noted accordingly in the text.

Cell culture and treatment. SV-HUC-1 cells were cultivated with F-12K growth medium with addition of $10 \% \mathrm{FBS}$, $100 \mathrm{U} / \mathrm{ml}$ penicillin and $100 \mu \mathrm{g} / \mathrm{ml}$ streptomycin under the atmosphere of $5 \% \mathrm{CO}_{2}$ at $37^{\circ} \mathrm{C}$ in an incubator. Cells were seeded in $10-\mathrm{cm}^{2}$ culture plates. The medium was changed every day until cells reached $80-90 \%$ confluence, and then was treated with various concentrations of benzidine, XMD8-92 or curcumin.
Cell toxicity assay. SV-HUC-1 cells were seeded in 96-well plate at the density of $2 \times 10^{3}$ cells/well. Then, the medium mixed with benzidine or curcumin at different concentrations was used to cultivate cells and changed every day for 4 days $(12,22,23)$. Ten microliters of methylthiazoletetrazolium assay solution $(5 \mathrm{mg} / \mathrm{ml})$ was added to each well and the plates were further incubated for $4 \mathrm{~h}$ at $37^{\circ} \mathrm{C}$. Medium containing MTT was removed and the precipitants were solubilized in DMSO. Absorbance was measured at $490 \mathrm{~nm}$ using a microplate reader (Titertek Instruments, Incorp., Huntsville, AL, USA). All measurements were performed in triplicate.

Migration assay. Wound healing assay was applied to detect change of migratory ability. Medium without benzidine or within benzidine at concentration of 0.005 or $0.05 \mu \mathrm{M}$ was used for pretreating SV-HUC-1 cells for 4 days. Then, cells were seeded in 6-well plate at $4 \times 10^{5} /$ well and cultivated for $24 \mathrm{~h}$ with serum-free medium before wounding. Afterwards, manual scrape was made to the cell monolayer to create a wound with a pipette tip. After washing the plate with PBS, each well was covered with medium to acquire images at the time-points of $0,12,24$ and $36 \mathrm{~h}$.

Invasion assay. The invasion assays were performed in a 24-well Boyden chamber with an $8-\mu \mathrm{m}$ pore size polycarbonate membrane (Millipore, Billerica, MA, USA) coated with Matrigel to form a matrix barrier. Pretreated cells without or with benzidine $(0.005$ and $0.05 \mu \mathrm{M})$ for 4 days, were suspended and $1 \times 10^{4}$ cells in $150 \mu \mathrm{l}$ serum-free medium were added to the upper compartment of the chamber. Moreover, $800 \mu \mathrm{l} \mathrm{F-12K}$ medium supplemented with $10 \%$ FBS was added into the lower compartment. After culturing in the incubator for $48 \mathrm{~h}$, cells remaining on the membranes inside the chamber were wiped off with a cotton-tipped swab, while cells adhered to lower membranes were fixed with methanol stained with crystal violet and finally photographed under a microscope. Then the number of invaded cells per microscope field was counted and analyzed.

Western blot analysis. SV-HUC-1 cells were harvest after a 4-day culture. Cells were washed with cryopreserved PBS and lysed with RIPA buffer (Thermo Fisher Scientific, Waltham, MA, USA). Concentrations of extracted proteins were measured with BCA protein assay (Pierce, Rockford, IL, USA). Then, the proteins were diluted to equal concentrations, boiled for $5 \mathrm{~min}$ and separated by 7.5-10\% SDS-PAGE, transferred onto polyvinylidene difluoride membranes (Millipore). Afterwards, membranes were blocked in 5\% fat-free dry milk in Tween-20 Tris-buffered saline (TBST) and then incubated in primary antibodies and secondary antibodies. Protein bands were subsequently developed using High-sig ECL western blotting substrate kit (Tanon Science and Technology, Co., Ltd., Shanghai, China). GAPDH served as loading control.

Quantitative real-time PCR. Total RNA was isolated by RNAiso Plus according to the manufacturer's instructions. (Takara Bio, Shiga, Japan). Then, total RNA was transcribed into cDNA using the AMV reverse transcriptase (Takara) following the manufacturer's protocol. qRT-PCR was performed using the Power SYBR-Green Master Mix (Takara) 
A

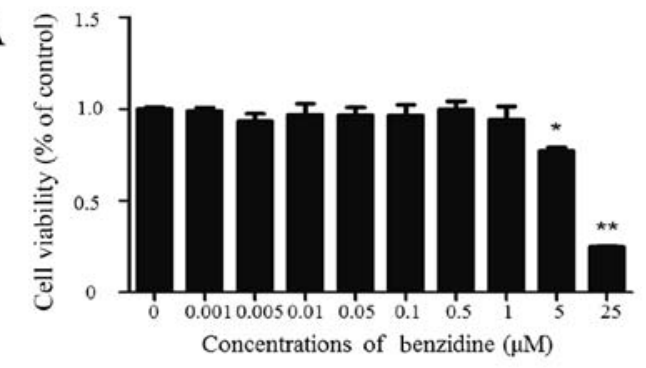

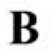

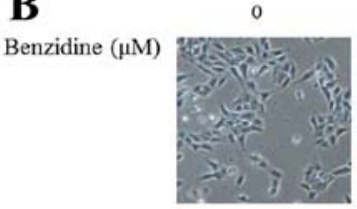

D

Benzidine $(\mu \mathrm{M})$
0

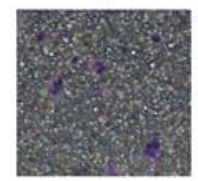

0.005

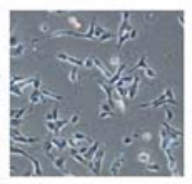

0.005

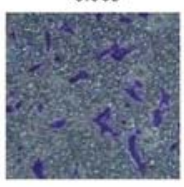

0.05

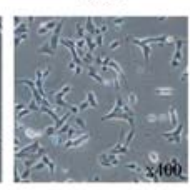

0.05

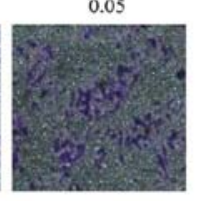

C B

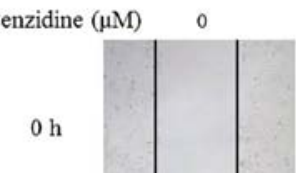
0.005

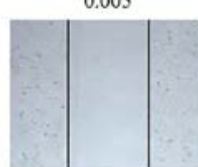

0.05

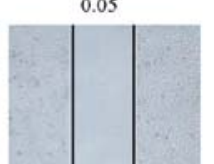

$12 \mathrm{~h}$
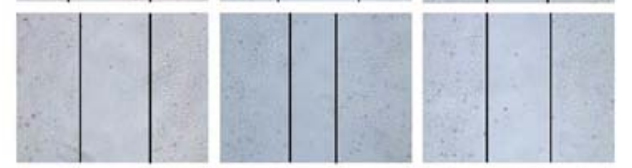

$24 \mathrm{~h}$
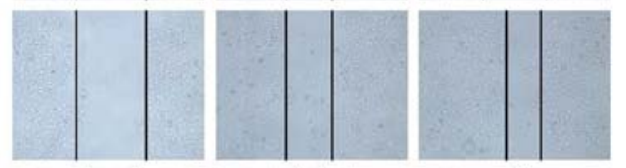

$36 \mathrm{~h}$
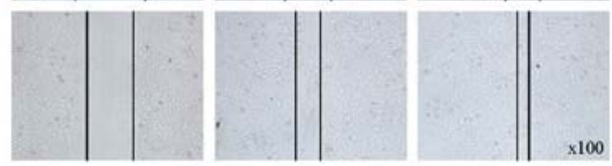

Figure 1. Benzidine exposure induces EMT in SV-HUC-1 cells. (A) MTT assay showed benzidine at concentrations $>25 \mu$ M weakened cell viability, while low concentrations showed no significant effect on cell viability. (B) Benzidine induced morphological change from epithelial to spinal-like mesenchymal shape. SV-HUC-1 cells were stretched to longer and thinner shape, part of which generated pseudopodium. (C) Wound healing assay revealed enhanced cell migratory capacity. (D) Invasive ability was reinforced as showed by the results of matrigel invasive assay and the numbers of cells were boosted significantly. Data are expressed as mean $\pm \mathrm{SD}$. ${ }^{*} \mathrm{P}<0.05,{ }^{* *} \mathrm{P}<0.01$.

and an ABI 7300 Real-Time PCR Detection system (Applied Biosystems, Foster City, CA, USA). The primers used were as follows: E-cadherin, forward, 5'-TCGACACCCGATTCAAA GTGG-3' and reverse, 5'-TTCCAGAAACGGAGGCCTG AT-3'; ZO-1, forward, 5'-GCAGCCACAACCAATTCATAG-3' and reverse, 5'-GCAGACGATGTTCATAGTTTC-3'; vimentin, forward, 5'-CCTTGACATTGAGATTGCCA-3' and reverse, 5'-GTATCAACCAGAGGGAGTGA-3'; N-cadherin forward, 5'-ATCAAGTGCCATTAGCCAAG-3' and reverse, 5'-CTGA GCAGTGAATGTTGTCA-3'; and GAPDH, forward, 5'-GCT GCCCAACGCACCGAATA-3' and reverse, 5'-GAGTCAAC GGATTTGGTCGT-3'. All of the primers were synthesized by Invitrogen (Carlsbad, CA, USA). The levels of mRNA expression for each gene were normalized by its respective GAPDH. Fold changes in gene expression were calculated by a comparative threshold cycle $(\mathrm{Ct})$ method using the formula $2^{-(\Delta \Delta \mathrm{Ct})}$.

Immunofluorescence. Pretreated SV-HUC-1 cells were transferred into 35-mm Glass bottom dishes (Cellvis, Mountain View, CA, USA) at the density of $5 \times 10^{4} /$ dish. After fixing and washing, dishes were stained with rabbit E-cadherin (1:150 dilution) and vimentin (1:100 dilution) antibodies at $4{ }^{\circ} \mathrm{C}$ overnight and then incubated with fluorescein isothiocyanate (FITC)-conjugated goat anti-rabbit secondary antibody for $1 \mathrm{~h}$. To stain the nuclei, 4',6-diamidino-2-phenylindole (DAPI; Sigma-Aldrich) was added for $10 \mathrm{~min}$. Images were captured using fluorescence microscopy (Zeiss, LSM700B; Carl Zeiss AG, Germany).

Statistical analysis. Statistical analyses were performed with the SPSS 16.0. All data were expressed as mean \pm standard deviation (SD). One-way ANOVA was used for comparison of statistical differences among multiple groups, followed by the LSD significant difference test. Unpaired Student's t-test was also used for the comparison between the two groups. $\mathrm{P}$-values of $\mathrm{P}<0.05$ or $\mathrm{P}<0.01$ were considered significantly different.

\section{Results}

Benzidine induces EMT in SV cells. Occupational exposure to aromatic amine is a major cause of bladder cancer, and benzidine-induced EMT is important in benzidine-associated malignant generation. To investigate the cell viability change in this process, cells were treated with benzidine $(0.001$, $0.005,0.01,0.05,0.1,1,5,25$ and $100 \mu \mathrm{M})$ for 4 days and were detected by MTT assay. The results showed that the cell viability decreased $<80 \%$ when the cells were exposed to $25 \mu \mathrm{M}$ benzidine or to higher concentrations (Fig. 1A). Therefore, concentrations range from 0.001 to $0.1 \mu \mathrm{M}$ were selected for the following experiments.

EMT process was assessed by alterations in the cell morphology, migratory and invasive capacity, and expression levels of epithelial and mesenchymal markers. Benzidine induced morphological change from epithelial to spindle-like mesenchymal shape, as shown by morphological examination of SV-HUC-1 cells following benzidine treatment for 4 days (Fig. 1B). Migratory ability was enhanced as wound healed in the treated groups compared with the control group (Fig. 1C). In invasion assay, cells transferred through the Matrigel at about the number of 10 per field in the control group. While in treated groups, cells increased to about 12 and 30 times, respectively, indicating bezidine induced elevated invasive capacity (Fig. 1D). 
A

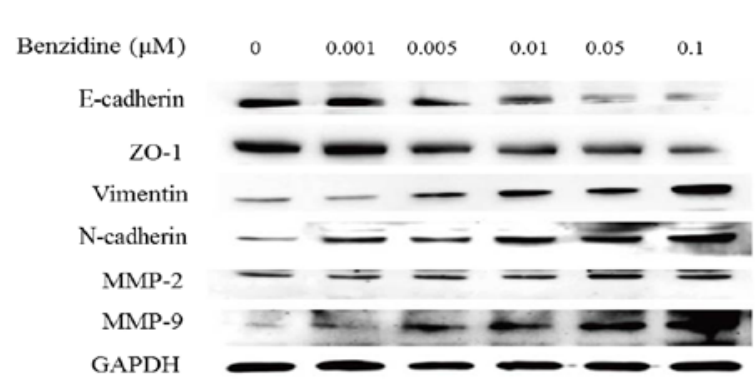

C

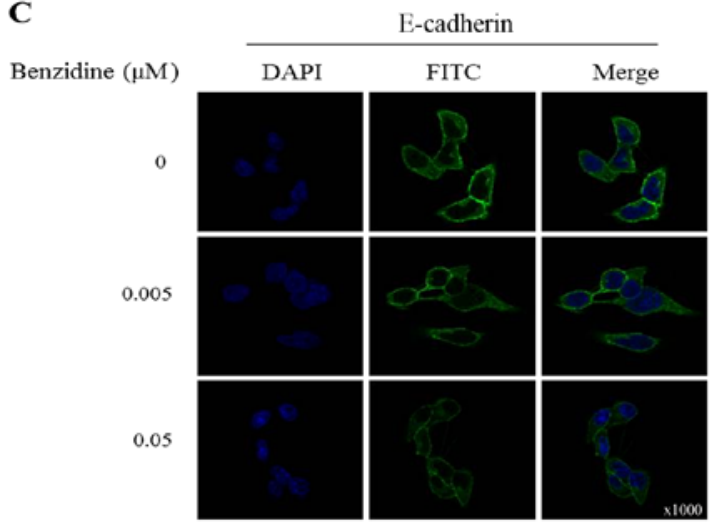

B

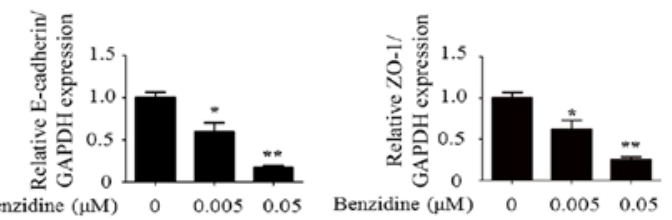

$\begin{array}{llllllll}\text { Benzidine }(\mu \mathrm{M}) & 0 & 0.005 & 0.05 & \text { Benzidine }(\mu \mathrm{M}) & 0 & 0.005 & 0.05\end{array}$
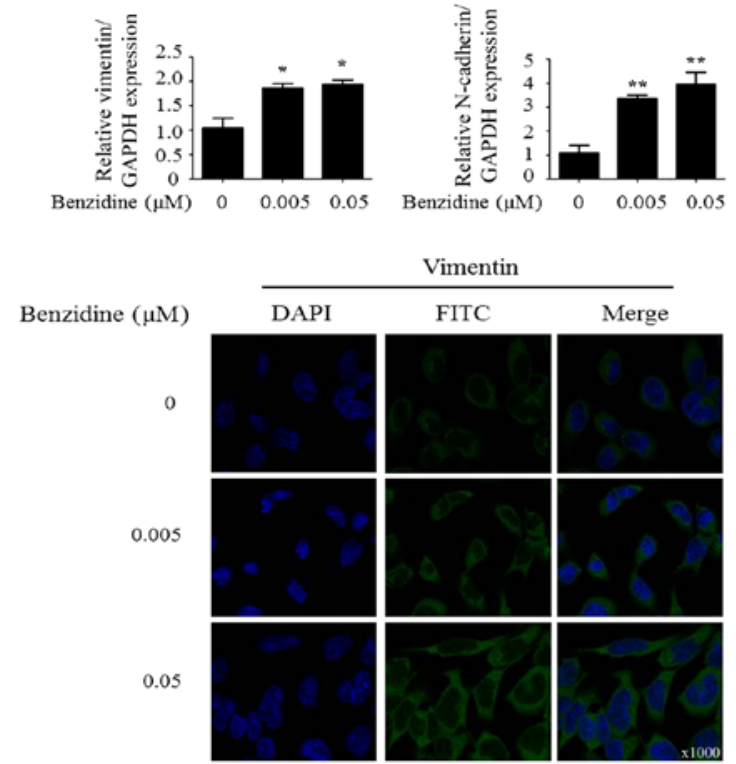

Figure 2. Benzidine alters the expression of EMT markers in SV-40 human immortalized human urothelial cells (SV-HUC-1). (A) Benzidine exposure downregulated protein levels of epithelial markers E-cadherin and ZO-1, upregulated protein levels of mesenchymal markers vimentin, N-cadherin, MMP-2 and MMP-9. (B) mRNA levels of E-cadherin and ZO-1 were decreased while mRNA levels of vimentin and N-cadherin were increased. (C) Immunofluorescent staining showed weakened protein level of E-cadherin and advanced protein level of vimentin with cell morphological changes. Data are expressed as mean \pm SD. ${ }^{*} \mathrm{P}<0.05,{ }^{* *} \mathrm{P}<0.01$.

A

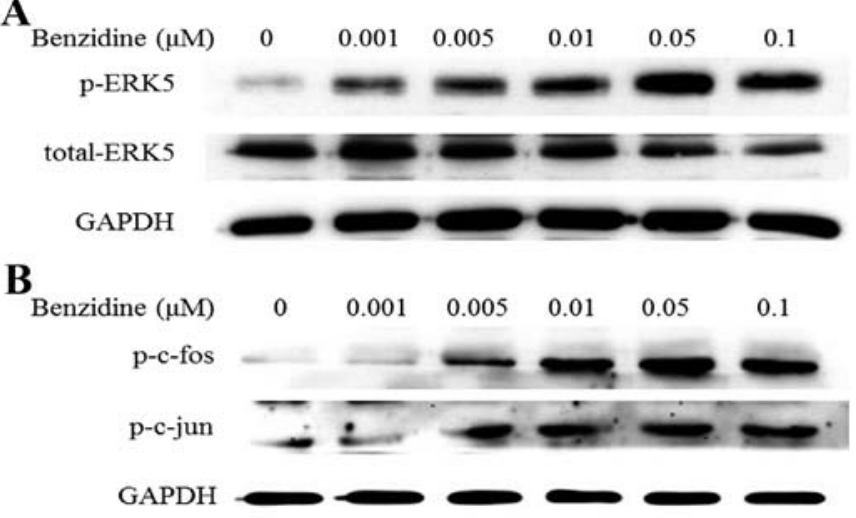

Figure 3. Benzidine exposure induces ERK5/AP-1 activation. (A) Treatment with benzidine induced ERK5 activation in SV-HUC-1 cells. The levels of phosphorylated ERK5, the active form of ERK5, as well as total ERK5, were measured by western blotting. (B) Benzidine increased AP-1 protein activation, as shown by elevated levels of phosphorylated c-Fos and phosphorylated c-Jun. GAPDH was used as loading control.

Occurrence of molecular changes. Western blot analysis exhibited the decrease of protein levels of epithelial markers E-cadherin and ZO-1. On the contrary, benzidine exposure enhanced the protein expression of mesenchymal markers vimentin, N-cadherin, MMP-2 and MMP-9 (Fig. 2A). Subsequent RT-PCT of mRNA levels of epithelial and mesenchymal markers showed similar changes. Expression levels of
E-cadherin mRNA at 0.005 and 0.05 were $55.3 \%(\mathrm{P}<0.05)$, $24.1 \%(\mathrm{P}<0.01)$. In addition, vimentin mRNA levels at 0.005 and 0.05 were $186.3 \%(\mathrm{P}<0.05)$ and $194.2 \%(\mathrm{P}<0.05)($ Fig. $2 \mathrm{~B})$. Results of immunofluorescent staining manifested changes not only in morphology but also in protein levels of epithelial and mesenchymal markers as described (Fig. 2C). Taken together, our results suggested that benzidine could induce EMT in SV-HUC-1 cells.

Benzidine activates ERK5/AP-1 signal pathway. ERK5 is a member of MAPKs family, which was thought to be oncosignaling. AP-1 is a downstream transcriptional factor of ERK5. To detect whether ERK5/AP-1 pathway was activated after SV-HUC-1 cells were exposed to benzidine for 4 days, protein levels of ERK5/AP-1 markers, p-ERK5, p-c-jun and p-c-fos were measured. The results showed notable increasing of p-ERK5 with dose-dependent manner and simultaneously restrained the level of total ERK5, indicating the upregulation effect of benzidine on ERK5 activity (Fig. 3A). Moreover, benzidine increased protein levels of $\mathrm{p}$-c-jun and $\mathrm{p}$-c-fos (Fig. 3B).

ERK5/AP-1 positively regulates benzidine-induced EMT. To further explore the correlation between benzidine-induced EMT and activation of ERK5/AP-1, XMD8-92 (5 $\mu \mathrm{M})$, a highly specific ERK5 inhibitor was used. Consistent with previous research, we found the inhibitor significantly suppressed the ERK5/AP-1 activity (Fig. 4A). Then we observed effects 


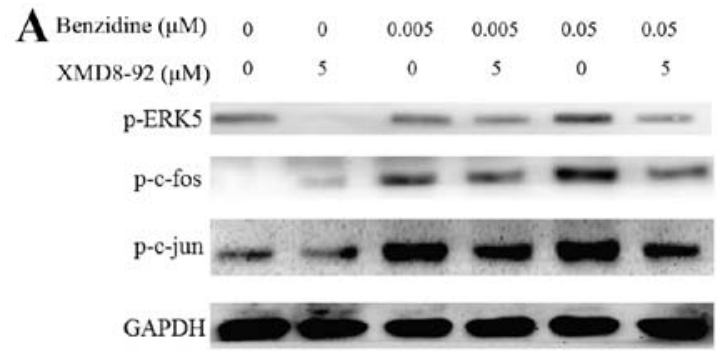

B

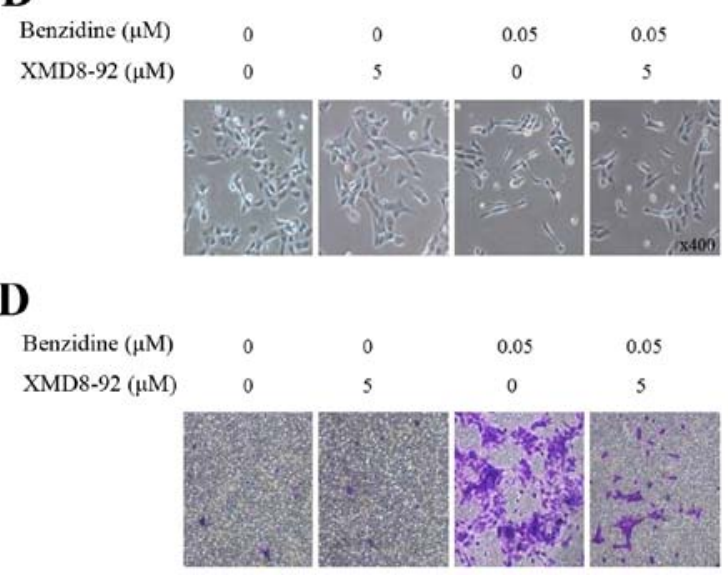

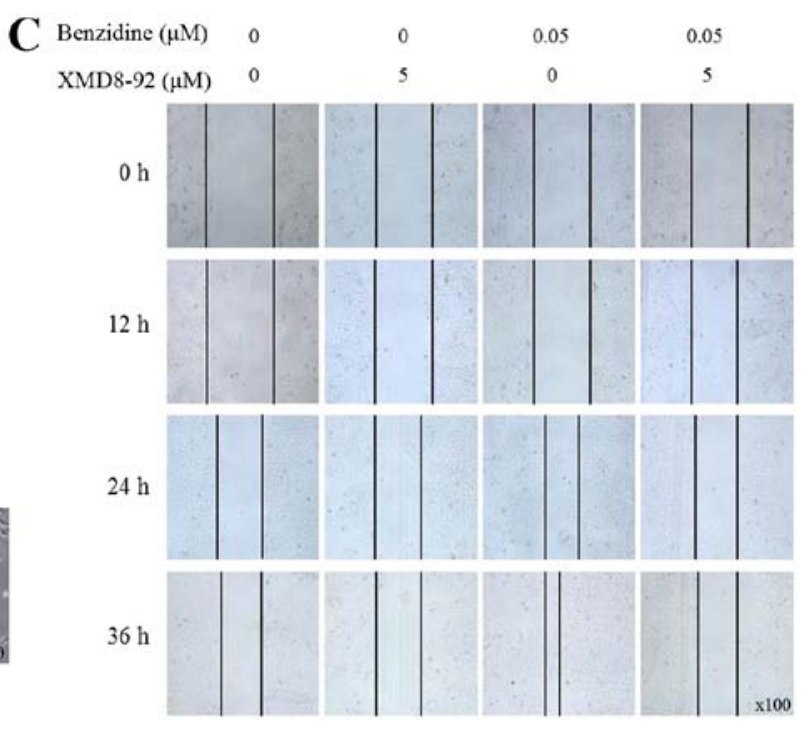

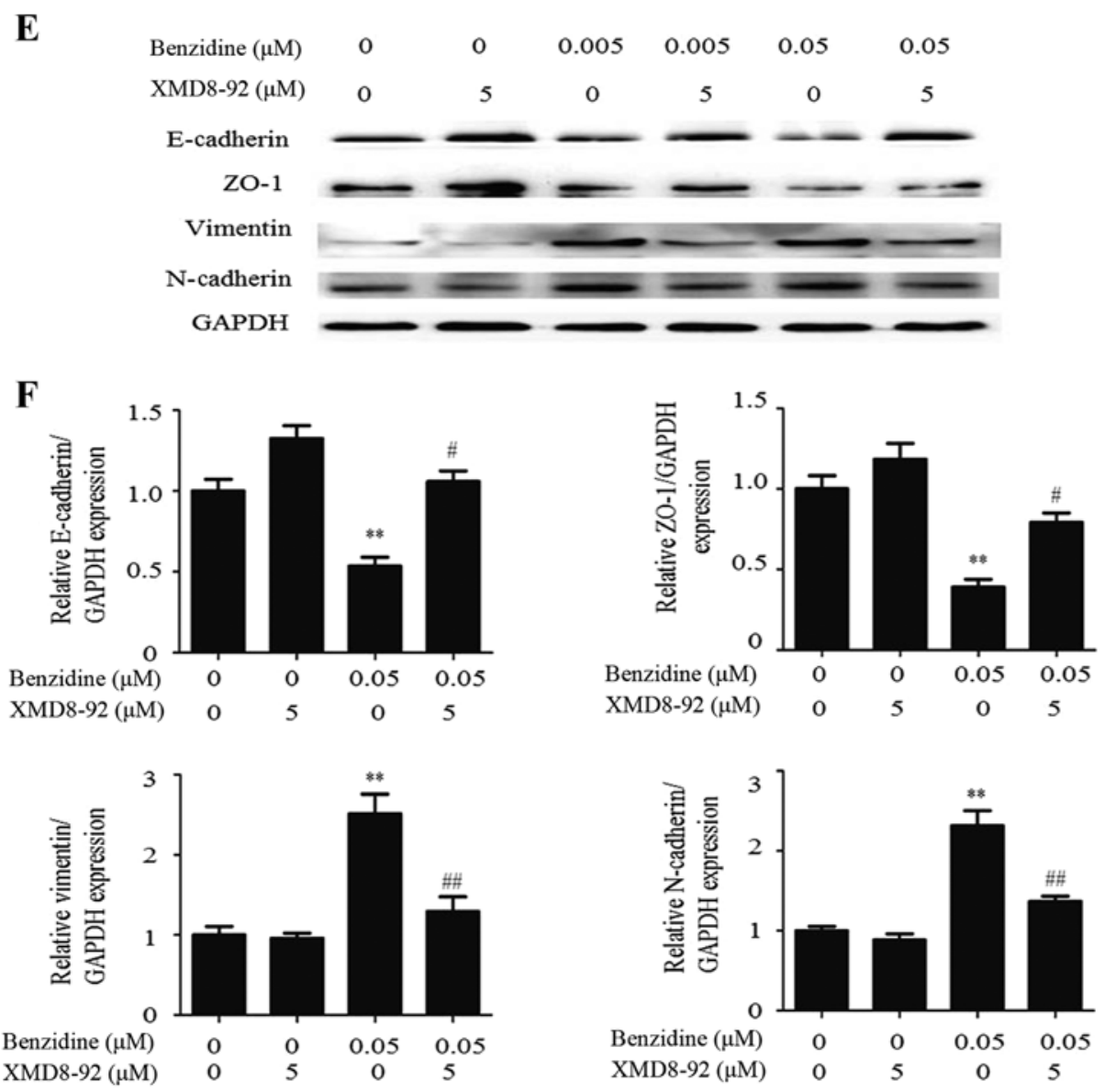

Figure 4. Inhibition of ERK5 attenuates benzidine-induced EMT in SV-HUC-1. (A) ERK5 inhibitor significantly depressed ERK5/AP-1 activity. SV-HUC-1 cells were treated with a highly specific inhibitor (XMD8-92) for 4 days, and western blot analyses were performed for the measurements of phosphorylated ERK5, phosphorylated c-Fos and phosphorylated c-Jun (B) ERK5 inhibitor reversed benzidine-provoked SV-HUC-1 morphological alterations. (C) Inhibition of ERK5 restrained benzidine-enhanced migratory ability in SV-HUC-1 cells as determined by wound healing assay. (D) Inhibition of ERK5 restrained benzidine-promoted invasive capacity as detected by Transwell invasion assay. (E) Cultivation with ERK5 inhibitor resulted in upregulated protein levels of E-cadherin and ZO-1 and downregulated protein levels of vimentin and N-cadherin. (F) Cultivation with ERK5 inhibitor increased the mRNA levels of E-cadherin and ZO-1 and decreased mRNA levels of vimentin and N-cadherin. Data are expressed as mean \pm SD. ${ }^{* *} \mathrm{P}<0.01$, benzidine groups with control groups. ${ }^{\#} \mathrm{P}<0.05,{ }^{\# \#} \mathrm{P}<0.01, \mathrm{XMD} 8-92$ groups with respective benzidine groups. 
A

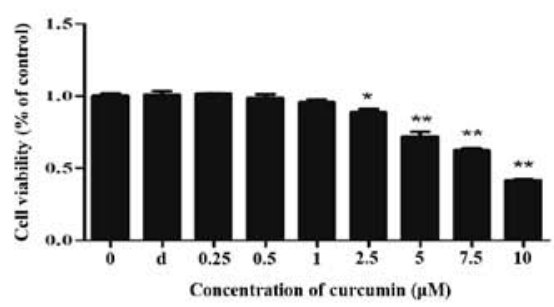

B

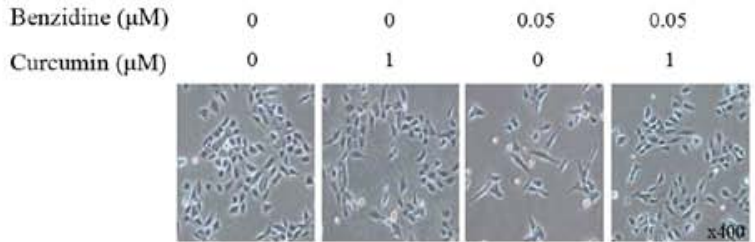

D

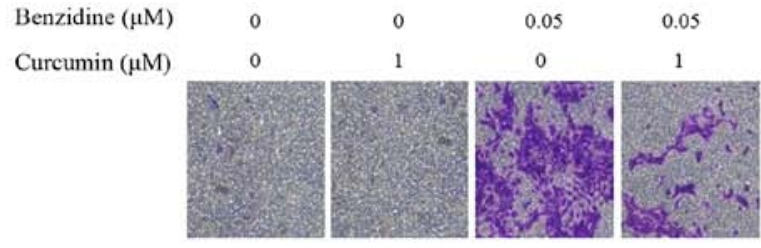

$\mathbf{E}$

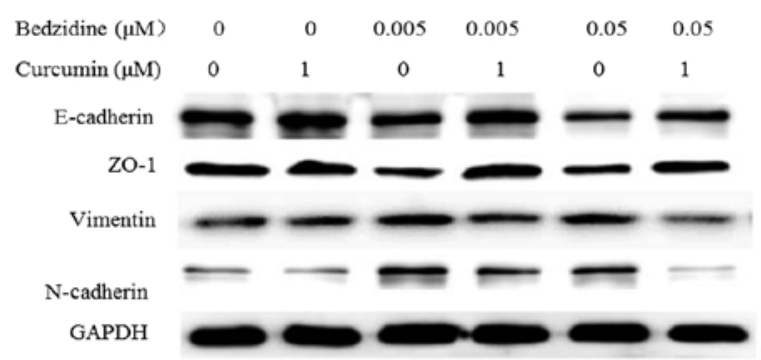

$\mathbf{F}$
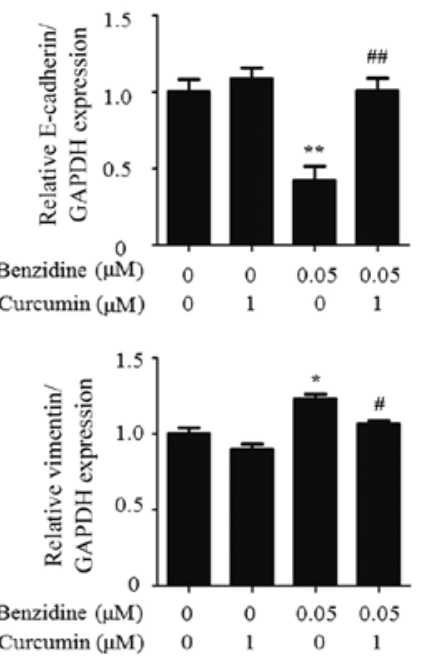

C

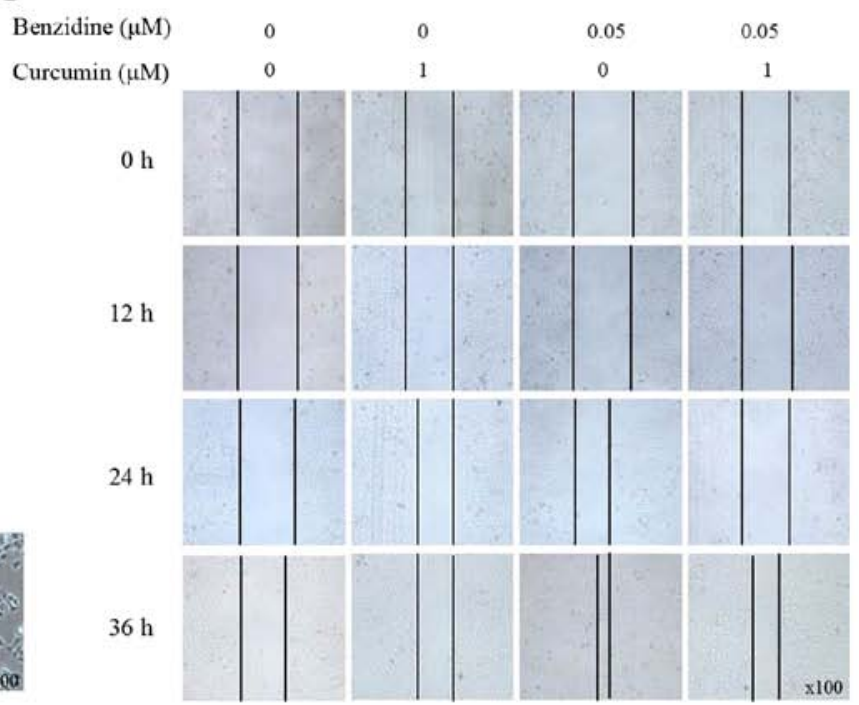

G

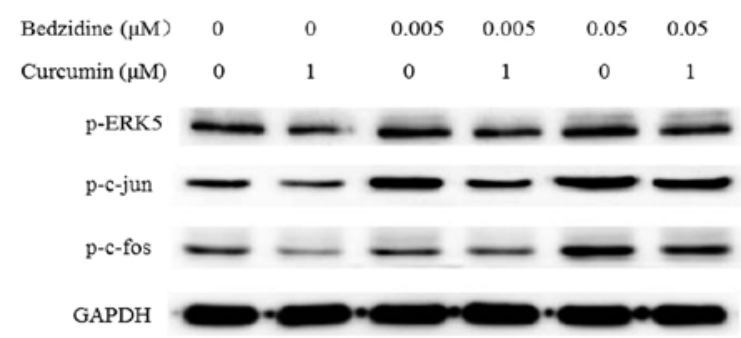

Curcumin $(\mu \mathrm{M}) \quad 0000505$

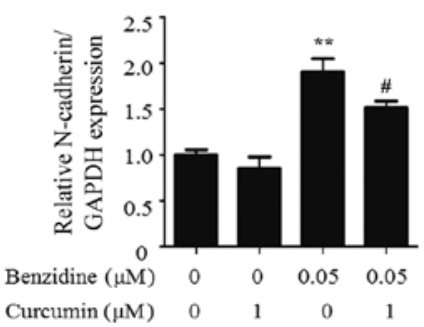

Figure 5. Curcumin repairs benzidine-induced SV-HUC-1 EMT via suppression of ERK5/AP-1. (A) Detection of cell viability after treating with curcumin for 4 days, curcumin at concentrations $<1 \mu \mathrm{M}$ had no impact on cell viability. Thus, dose of $1 \mu \mathrm{M}$ was chosen for consequent experiments. (B) Curcumin attenuated benzidine-induced cell morphology changes. (C) Curcumin reversed benzidine-elevated cell migratory capacity. (D) Treatment with benzidine weakened benzidine-enhanced cell invasive ability as detected by Transwell invasive assay. (E) Cultivation with curcumin leads to elevation of expression levels of E-cadherin and ZO-1 as well as decreased expression levels of vimentin and $\mathrm{N}$-cadherin as determined by western blotting. (F) Cultivation with curcumin upregulated mRNA levels of E-cadherin and ZO-1 and downregulated vimentin and N-cadherin mRNA levels. (G) Curcumin suppressed ERK5/AP-1 activity. Benzidine-induced elevation of phosphorylated ERK5, phosphorylated c-Fos and phosphorylated c-Jun was reversed by curcumin. Data are expressed as mean $\pm \mathrm{SD} .{ }^{* *} \mathrm{P}<0.01$, benzidine groups with control groups. ${ }^{\#} \mathrm{P}<0.05,{ }^{\# \#} \mathrm{P}<0.01$, curcumin groups with respective benzidine groups. 
of XMD8-92 on benzidine-induced EMT process. Results showed that inhibitor alone had no effect on cell phenotype, while benzidine-induced SV-HUC-1 morphological changes were reversed after cells were exposed to benzidine combined with the inhibitor (Fig. 4B). Inhibitor resulted in attenuation of benzidine-enhanced cell migration and invasion ability (Fig. 4C and D).

Moreover, molecular changes were detected. Results indicated that suppression of ERK 5 activity leads to downregulation of epithelial markers E-cadherin, ZO-1 as well as upregulation of mesenchymal markers vimentin and $\mathrm{N}$-cadherin in protein and mRNA levels (Fig. 4E and F). Collectively, our results indicated that benzidine promoted SV-HUC-1 EMT via upregulating the ERK5/AP-1 signal pathway.

Curcumin attenuates benzidine-induced EMT via suppression of ERK5/AP-1. To determine whether curcumin could interfere with benzidine-induced EMT, we first conducted MTT assay to screen appropriate concentration for subsequent experiments. The data showed that curcumin at doses $<1 \mu \mathrm{M}$ had no influence on cell viability, and cell viability was decreased $<85 \%$ at concentrations $>2.5 \mu \mathrm{M}$ and had significance (Fig. 5A). Therefore, curcumin at $1 \mu \mathrm{M}$ was chosen for the following experiments. Results showed that curcumin alone had no effect on cell morphology. The benzidine-mediated cell morphological changes were reversed by curcumin (Fig. 5B). Benzidine-enhanced capacity of migration and invasion were also detected to be weakened by curcumin (Fig. 5C and D).

Subsequently, we measured epithelium and mesenchymal markers at molecular levels. Results indicated that treatment with curcumin elevated protein and mRNA levels of E-cadherin and ZO-1, reduced vimentin and $\mathrm{N}$-cadherin, which demonstrated the suppression of curcumin in benzidine-induced EMT (Fig. 5E and F). Furthermore, we detected ERK5/AP-1 activity as cells exposed to curcumin and found that benzidineactivated ERK5/AP-1 was inhibited by curcumin (Fig. 5G). In summary, these findings confirmed that curcumin was able to reverse benzidine-triggered SV-HUC-1 cell EMT via suppression of the ERK5/AP-1 signal pathway.

\section{Discussion}

Occupational exposure to aromatic amine including benzidine has been manifested as a major cause of bladder cancer. However, the underlying mechanisms by which benzidine results in tumorigenesis remain to be elucidated. Results of a previous study revealed the important role of ERK $1 / 2$ in benzidine-triggered EMT (12), whereas the role of ERK5 in benzidine-promoted bladder cancer development has not been investigated. In addition, the chemical prevention of benzidine-induced malignancy has not been explored. In the present study, we demonstrated the regulation role of ERK5/AP-1 in benzidine-induced EMT in SV-HUC-1 cells. Moreover, we identified the prevention effect of curcumin on benzidine-mediated EMT. These finding may provide new insight into benzidine-related tumor formation, as well as chemical intervention for bladder cancer.

Characteristics by alteration in cell morphology, migratory and invasive capacity, as well as expression of epithelial and mesenchymal markers, EMT is considered as a critical step for the initiation and development of epithelium-originated malignancy. It has been documented that many molecular changes occur during EMT process, including downregulation of E-cadherin and upregulation of $\mathrm{N}$-cadherin. E-cadherin has been established as a cell adhesion molecule, which plays an essential role in epithelial cell-to-cell interactions since it mediates the connections between adjacent epithelial cells and maintains the phenotype and apical-base polarity of epithelial cells. N-cadherin (neural cadherin), another adhesion molecule, is associated with a heightened migratory and invasive potential in cancer $(24,25)$. Consistent with former studies, the results of the present study showed that benzidine exposure induces EMT in SV-HUV-1 cell line manifested by the cell phenotype and molecular changes after benzidine exposure. Taken together, we identified benzidine induced EMT in vitro.

Several signal pathways have been implicated in EMT, such as PI3K, NF- $\kappa$ B and MAPK (26-28). To date, scarce research has been conducted to explore the function of ERK5 in benzidine-triggered EMT. In the present study, we found that ERK5 was activated during benzidine exposure. We further confirmed the regulation of ERK5 in urocystic EMT. ERK5 inhibitor significantly reversed benzidine-induced EMT, as showed by repaired cell shape, attenuated migratory and invasive capacity and molecular changes. In summary, these results indicated the upregulation role of ERK5 in benzidine-induced EMT.

ERK5 is twice the size of the other members. Similar to ERK1/2, the N-terminal of ERK5 contains a kinase domain and has the Thr-Glu-Tyr (TEY) activation motif. ERK5 differ in its large C-terminal-half that contains a transcriptional activation domain. Upon activation, ERK5 could not only phosphorylate and activate downstream target molecules, including transcription factors such as members of the AP-1 proteins, but also auto-phosphorylate C-terminus, which alone has the ability to increase transcriptional activity (29-31). Therefore, ERK5 is different from other ERKs for possessing transcriptional activation activity. Given its unique structure, ERK5 regulates the transcription of downstream molecules in two ways i.e., through either the phosphorylation or the enhancement of the transcription activity of target molecules $(14,15)$. It has been found that ERK5 transcriptionally regulates gene expression in a tissue-specific manner (32). In breast cancer cells, activation of ERK5 significantly enhance cyclin D1 expression and promotes neoplastic transformation (33). In lung microvascular endothelial cells, activation of ERK 5 suppresses HIF-1 $\alpha$ expression and inhibits angiogenesis (34). In this study, we showed that benzidine-meditated activation of ERK5 triggered the activation of AP-1 proteins c-Jun and c-Fos; on the contrary, inhibition of ERK5 suppressed the activation of c-Jun and c-Fos, indicating the positive modulation role of ERK 5 on AP-1. Research has proved the important role of AP-1 proteins in EMT modulation by elevating EMT-related molecules and transcriptional factors such as Zeb1/2, TGF $\beta$, Twist1, integrin $\alpha 5$ and epithelial cell adhesion molecule (EpCAM), which in turn transcriptionally regulate expression of mesenchymal markers vimentin and $\mathrm{N}$-cadherin, and epithelial markers E-cadherin and ZO-1 (35-38). In the present study, we found that benzidine activated ERK5, leading to activation of AP-1, resulting in promoted migratory 
and invasive abilities, as well as decreased levels of epithelial markers and increased levels of mesenchymal markers. In summary, the present study demonstrated the positive role of ERK5/AP-1 in benzidine-induced urocystic EMT.

As a promising natural compound, curcumin has been applied to cancer therapy. Curcumin pleiotropic activities emanate from its ability to modulate numerous signaling, transcriptional molecules, signal transducer and adhesion molecules such as MMP-9, intracellular adhesion molecule-1 (ICAM-1) and vascular cell adhesion molecule-1 (VCAM-1) $(39,40)$. Evidence has confirmed that curcumin regulates different signal pathways or cytokines to prevent cancer initiation and promotion. It has been acknowledged that curcumin can be used as an efficient adjuvant to cisplatin cancer therapy by modulating STAT3 and Nrf2 signaling in human squamous carcinoma (41). Woo et al (42) demonstrated that curcumin suppresses MAPK signaling pathways in human astroglioma cells. As for the present study, we proved that curcumin repairs benzidine-induced EMT in both phenotype and genotype levels. Moreover, benzidine-triggered activation of ERK5/AP-1 signal pathway was attenuated by curcumin, indicating the interventional role of curcumin in benzidinemeditated urocystic malignancies.

In conclusion, this study identified the positive role of ERK5 in benzidine-promoted EMT, as well as the suppression role of curcumin in benzidine-related bladder malignancy. These findings indicate new mechanism of benzidine-induced bladder malignancy and provide a potential strategy for prevention and intervention of bladder cancer.

\section{Acknowledgements}

The present study was supported by grants from the National Natural Science Foundation of China (nos. 81373005, 81072330 and 81202194), the Priority Academic Program Development of Jiangsu Higher Education Institutions (Public Health and Preventive Medicine), the Anhui Medical University Scientific Research Funds (Hefei, China; grant no. H0514) and the Anhui Public Welfare Research Linkage Plan (Hefei, China; grant no. 1501ld04045).

\section{References}

1. Chen W, Zheng R, Baade PD, Zhang S, Zeng H, Bray F, Jemal A, $\mathrm{Yu}$ XQ and He J: Cancer statistics in China, 2015. CA Cancer J Clin 66: 115-132, 2016.

2. Letašiová S, Medve'ová A, Šovčíková A, Dušinská M, Volkovová K, Mosoiu C and Bartonová A: Bladder cancer, a review of the environmental risk factors. Environ Health 11 (Suppl 1): S11, 2012.

3. Choudhary G: Human health perspectives on environmental exposure to benzidine: A review. Chemosphere 32: 267-291, 1996.

4. Whysner J, Verna L and Williams GM: Benzidine mechanistic data and risk assessment: Species- and organ-specific metabolic activation. Pharmacol Ther 71: 107-126, 1996.

5. Baan R, Grosse Y, Straif K, Secretan B, El Ghissassi F, Bouvard V, Benbrahim-Tallaa L, Guha N, Freeman C, Galichet L, et al; WHO International Agency for Research on Cancer Monograph Working Group: A review of human carcinogens--Part F: Chemical agents and related occupations. Lancet Oncol 10 $1143-1144,2009$.

6. Snyderwine EG, Sinha R, Felton JS and Ferguson LR: Highlights of the eighth international conference on carcinogenic/mutagenic $\mathrm{N}$-substituted aryl compounds. Mutat Res 506-507: 1-8, 2002.
7. Yu MC, Skipper PL, Tannenbaum SR, Chan KK and Ross RK: Arylamine exposures and bladder cancer risk. Mutat Res 506-507: 21-28, 2002.

8. Chen HI, Liou SH, Loh CH, Uang SN, Yu YC and Shih TS: Bladder cancer screening and monitoring of 4,4'-methylenebis(2chloroaniline) exposure among workers in Taiwan. Urology 66: 305-310, 2005.

9. Kalluri R: EMT: When epithelial cells decide to become mesenchymal-like cells. J Clin Invest 119: 1417-1419, 2009.

10. $\mathrm{Li} \mathrm{L}$ and $\mathrm{Li} \mathrm{W}$ : Epithelial-mesenchymal transition in human cancer: Comprehensive reprogramming of metabolism, epigenetics, and differentiation. Pharmacol Ther 150: 33-46, 2015.

11. Voulgari A and Pintzas A: Epithelial-mesenchymal transition in cancer metastasis: Mechanisms, markers and strategies to overcome drug resistance in the clinic. Biochim Biophys Acta 1796: 75-90, 2009.

12. Zhao L, Geng H, Liang ZF, Zhang ZQ, Zhang T, Yu DX and Zhong CY: Benzidine induces epithelial-mesenchymal transition in human uroepithelial cells through ERK1/2 pathway. Biochem Biophys Res Commun 459: 643-649, 2015.

13. Chang L and Karin M: Mammalian MAP kinase signalling cascades. Nature 410: 37-40, 2001

14. Drew BA, Burow ME and Beckman BS: MEK5/ERK5 pathway: The first fifteen years. Biochim Biophys Acta 1825: 37-48, 2012.

15. Nishimoto $\mathrm{S}$ and Nishida E: MAPK signalling: ERK5 versus ERK1/2. EMBO Rep 7: 782-786, 2006.

16. Hayashi M, Fearns C, Eliceiri B, Yang Y and Lee JD: Big mitogen-activated protein kinase 1/extracellular signal-regulated kinase 5 signaling pathway is essential for tumor-associated angiogenesis. Cancer Res 65: 7699-7706, 2005.

17. Amano S, Chang YT and Fukui Y: ERK5 activation is essential for osteoclast differentiation. PLoS One 10: e0125054, 2015.

18. Zhong CY,Zhou YM, Douglas GC, Witschi H and Pinkerton KE: MAPK/AP-1 signal pathway in tobacco smoke-induced cell proliferation and squamous metaplasia in the lungs of rats. Carcinogenesis 26: 2187-2195, 2005.

19. Zhao J, Harper R, Barchowsky A and Di YPP: Identification of multiple MAPK-mediated transcription factors regulated by tobacco smoke in airway epithelial cells. Am J Physiol Lung Cell Mol Physiol 293: L480-L490, 2007.

20. Geng H, Zhao L, Liang Z, Zhang Z, Xie D, BiL, Wang Y, Zhang T, Cheng L, Yu D, et al: ERK5 positively regulates cigarette smokeinduced urocystic epithelial-mesenchymal transition in SV-40 immortalized human urothelial cells. Oncol Rep 34: 1581-1588, 2015.

21. Park W, Amin ARMR, Chen ZG and Shin DM: New perspectives of curcumin in cancer prevention. Cancer Prev Res (Phila) 6: 387-400, 2013

22. Kawami M, Harabayashi R, Miyamoto M, Harada R, Yumoto R and Takano M: Methotrexate-induced epithelial-mesenchymal transition in the alveolar epithelial cell line A549. Lung 194: 923-930, 2016.

23. Zhang P, Dong Z, Cai J, Zhang C, Shen Z, Ke A, Gao D, Fan J and Shi G: BRD4 promotes tumor growth and epithelialmesenchymal transition in hepatocellular carcinoma. Int J Immunopathol Pharmacol 28: 36-44, 2015.

24. Peralta Soler A, Knudsen KA, Jaurand MC, Johnson KR Wheelock MJ, Klein-Szanto AJ and Salazar H: The differential expression of $\mathrm{N}$-cadherin and E-cadherin distinguishes pleural mesotheliomas from lung adenocarcinomas. Hum Pathol 26: 1363-1369, 1995.

25. Nakajima S, Doi R, Toyoda E, Tsuji S, Wada M, Koizumi M, Tulachan SS, Ito D, Kami K, Mori T, et al: N-cadherin expression and epithelial-mesenchymal transition in pancreatic carcinoma. Clin Cancer Res 10: 4125-4133, 2004.

26. Zhang X, Song Q, Wei C and Qu J: LRIG1 inhibits hypoxiainduced vasculogenic mimicry formation via suppression of the EGFR/PI3K/AKT pathway and epithelial-to-mesenchymal transition in human glioma SHG-44 cells. Cell Stress Chaperones 20: 631-641, 2015.

27. Li J, Deng Z, Wang Z, Wang D, Zhang L, Su Q, Lai Y, Li B, Luo Z, Chen X, et al: Zipper-interacting protein kinase promotes epithelial-mesenchymal transition, invasion and metastasis through AKT and NF- $\mathrm{NB}$ signaling and is associated with metastasis and poor prognosis in gastric cancer patients. Oncotarget 6: 8323-8338, 2015. 
28. Park JH, Yoon J, Lee KY and Park B: Effects of geniposide on hepatocytes undergoing epithelial-mesenchymal transition in hepatic fibrosis by targeting TGF $\beta /$ Smad and ERK-MAPK signaling pathways. Biochimie 113: 26-34, 2015.

29. Kasler HG, Victoria J, Duramad O and Winoto A: ERK5 is a novel type of mitogen-activated protein kinase containing a transcriptional activation domain. Mol Cell Biol 20: 8382-8389, 2000.

30. Morimoto H, Kondoh K, Nishimoto S, Terasawa K and Nishida E: Activation of a C-terminal transcriptional activation domain of ERK5 by autophosphorylation. J Biol Chem 282: 35449-35456, 2007.

31. Nithianandarajah-Jones GN, Wilm B, Goldring CE, Müller J and Cross MJ: ERK5: Structure, regulation and function. Cell Signal 24: 2187-2196, 2012

32. Sohn SJ, Li D, Lee LK and Winoto A: Transcriptional regulation of tissue-specific genes by the ERK 5 mitogen-activated protein kinase. Mol Cell Biol 25: 8553-8566, 2005.

33. Mulloy R, Salinas S, Philips A and Hipskind RA: Activation of cyclin D1 expression by the ERK5 cascade. Oncogene 22: $5387-5398,2003$

34. Pi X, Garin G, Xie L, Zheng Q, Wei H, Abe J, Yan C and Berk BC: BMK1/ERK5 is a novel regulator of angiogenesis by destabilizing hypoxia inducible factor 1alpha. Circ Res 96: 1145-1151, 2005

35. Bakiri L, Macho-Maschler S, Custic I, Niemiec J, GuíoCarrión A, Hasenfuss SC, Eger A, Müller M, Beug $\mathrm{H}$ and Wagner EF: Fra-1/AP-1 induces EMT in mammary epithelial cells by modulating Zeb1/2 and TGF $\beta$ expression. Cell Death Differ 22: 336-350, 2015

36. Qiao Y, Shiue CN, Zhu J, Zhuang T, Jonsson P, Wright AP, Zhao $\mathrm{C}$ and Dahlman-Wright K: AP-1-mediated chromatin looping regulates ZEB2 transcription: New insights into $\mathrm{TNF} \alpha-$ induced epithelial-mesenchymal transition in triple-negative breast cancer. Oncotarget 6: 7804-7814, 2015.
37. Nam EH, Lee Y, Moon B, Lee JW and Kim S: Twist1 and AP-1 cooperatively upregulate integrin $\alpha 5$ expression to induce invasion and the epithelial-mesenchymal transition. Carcinogenesis 36: 327-337, 2015.

38. Gao J, Yan Q, Wang J, Liu S and Yang X: Epithelial-tomesenchymal transition induced by TGF- $\beta 1$ is mediated by AP1-dependent EpCAM expression in MCF-7 cells. J Cell Physiol 230: 775-782, 2015.

39. Gupta SC, Patchva S and Aggarwal BB: Therapeutic roles of curcumin: Lessons learned from clinical trials. AAPS J 15: 195-218, 2013.

40. Shanmugam MK, Rane G, Kanchi MM, Arfuso F, Chinnathambi A, Zayed ME, Alharbi SA, Tan BK, Kumar AP and Sethi G: The multifaceted role of curcumin in cancer prevention and treatment. Molecules 20: 2728-2769, 2015.

41. Fetoni AR, Paciello F, Mezzogori D, Rolesi R, Eramo SL, Paludetti G and Troiani D: Molecular targets for anticancer redox chemotherapy and cisplatin-induced ototoxicity: The role of curcumin on pSTAT3 and Nrf-2 signalling. Br J Cancer 113: 1434-1444, 2015 .

42. Woo MS, Jung SH, Kim SY, Hyun JW, Ko KH, Kim WK and Kim HS: Curcumin suppresses phorbol ester-induced matrix metalloproteinase-9 expression by inhibiting the PKC to MAPK signaling pathways in human astroglioma cells. Biochem Biophys Res Commun 335: 1017-1025, 2005. 